\title{
Quotients of Banach Spaces with the Daugavet Property
}

by

\author{
Vladimir KADETS, Varvara SHEPELSKA and Dirk WERNER
}

Presented by Aleksander PEŁCZYŃSKI

Summary. We consider a general concept of Daugavet property with respect to a norming subspace. This concept covers both the usual Daugavet property and its weak* analogue. We introduce and study analogues of narrow operators and rich subspaces in this general setting and apply the results to show that a quotient of $L_{1}[0,1]$ by an $\ell_{1}$-subspace need not have the Daugavet property. The latter answers in the negative a question posed to us by A. Pełczyński.

1. Introduction. Throughout the paper $X$ stands for a Banach space. Recall that $X$ has the Daugavet property if the identity

$$
\|\mathrm{Id}+T\|=1+\|T\|,
$$

called the Daugavet equation, holds true for every rank-one operator $T: X$ $\rightarrow X$. (We shall find it convenient to abbreviate this by writing $X \in \mathrm{DPr}$.) It is known that in this case (1.1) holds for the much wider class of so-called narrow operators. This class includes all strong Radon-Nikodým operators (which map the unit ball into a set with the Radon-Nikodým property) and in particular compact and weakly compact operators, operators not fixing a copy of $\ell_{1}$, and linear combinations of the above mentioned types of operators [10].

Among the spaces with the Daugavet property are $C(K)$-spaces and vector-valued $C(K)$-spaces for perfect compact Hausdorff spaces $K, L_{1}(\mu)$ -

2000 Mathematics Subject Classification: Primary 46B04; Secondary 46B03, 46B25, $47 \mathrm{~B} 38$.

Key words and phrases: Daugavet property, Daugavet equation, rich subspace, poor subspace, narrow operator, Radon-Nikodým property.

Research of the first named author was partially supported by a grant from the Alexander-von-Humboldt Foundation. 
and vector-valued $L_{1}(\mu)$-spaces for non-atomic $\mu$, wide classes of Banach algebras, but also some exotic spaces like Talagrand's space from [14] (see [9]) or Bourgain-Rosenthal's space from [3] (see [11]). All the spaces with the Daugavet property are non-reflexive; moreover, they cannot have the Radon-Nikodým property and necessarily contain "many" copies of $\ell_{1}$ [9].

There are several results on the stability of the Daugavet property under passing to "big" subspaces or quotients by "small" subspaces. In particular, if $X \in \mathrm{DPr}$, then $X / E \in \mathrm{DPr}$ for every reflexive subspace $E \subset X$ [13]. A preliminary version of that theorem appeared in [9] for $X=L_{1}[0,1]$. In a private conversation after a talk by the third-named author on the results of [9], A. Pełczyński asked whether the last result can be generalized to $E \subset L_{1}[0,1]$ that are not necessarily reflexive, but have the Radon-Nikodým property (RNP). The question appeared quite non-trivial for the authors of [9], maybe because the efforts were concentrated on attempts to prove that $L_{1}[0,1] / E \in \mathrm{DPr}$ if $E$ has the RNP. This question was reiterated in [13] and [7]. In this paper we finally present a negative answer.

Our approach to Pełczyński's question will be indirect. If the answer were positive, this would mean that every subspace $E \subset L_{1}[0,1]$ with the RNP has the following "smallness" property, since the RNP is hereditary: $L_{1}[0,1] / F \in \mathrm{DPr}$ for every subspace $F \subset E$. We introduce this property formally in a general setting (we call it poverty) and characterise it geometrically. Then we give a description of poor subspaces of $L_{1}(\Omega, \Sigma, \mu)$, and using that description we present an $\ell_{1}$-subspace $E$ of $L_{1}[0,1]$ which is not poor. Since $\ell_{1}$ has the RNP, this leads to a counterexample.

To do all this we use duality arguments, but in order to be able to apply these arguments we have to consider a generalisation of the Daugavet property. Let us recall that a subspace $Y \subset X^{*}$ is said to be norming (more precisely 1-norming) if

$$
\|x\|=\sup \left\{\left|y^{*}(x)\right|: y^{*} \in Y,\left\|y^{*}\right\| \leq 1\right\}
$$

for every $x \in X$. This is equivalent to saying that the closed unit ball of $Y$ is weak* dense in the closed unit ball of $X^{*}$. Throughout the paper $Y$ denotes a norming subspace of $X^{*}$.

Definition 1.1. We say that $X$ has the Daugavet property with respect to $Y(X \in \operatorname{DPr}(Y))$ if the Daugavet equation (1.1) holds true for every rank-one operator $T: X \rightarrow X$ of the form $T=y^{*} \otimes x$, where $x \in X$ and $y^{*} \in Y$.

This generalisation of the ordinary Daugavet property was introduced in an equivalent form in [1]. It was motivated by the fact that the Daugavet property is not stable under passing to ultraproducts (this was proved in [11], but was an open problem at the time when [1] was written), but the ul- 
traproduct of spaces with the Daugavet property has the Daugavet property with respect to the ultraproduct of the dual spaces. The basic motivation for us in the present paper is that the Daugavet property does not generally pass to the dual, but it is obvious that if the original space $X$ has the Daugavet property, then $X^{*} \in \operatorname{DPr}(X)$.

The structure of the paper is as follows. Section 2 contains characterisations of the Daugavet property with respect to $Y$ in terms of slices, similar to [9]. Our eventual aim is to study "small" subspaces $Z \subset X$ of spaces with the Daugavet property, called poor subspaces; this will be done in Section 5 . It will turn out that $Z \subset X$ is poor if and only if $Z^{\perp} \subset X^{*}$ enjoys a variant of the properties called richness and wealth in [10]. Such spaces are defined by means of a smallness property of the corresponding quotient map called narrowness. Narrow operators are studied in our context in Section 4. In order to prove that weakly compact operators on dual spaces are $X$-narrow we have included the technical Section 3 about convex combinations of slices. Finally, in Section 6 we characterise poor subspaces of $C(K)$ and $L_{1}(\mu)$ and derive that both spaces contain copies of $\ell_{1}$ that are not poor provided they are separable, $K$ is perfect and $\mu$ is atomless. This leads to a negative answer to Pełczyński's question mentioned above (see Theorem 6.10).

A reader who is interested in that result only might wish to skip Section 3 and Section 4 apart from Definition 4.3, and he or she might also wish to only consider the case $X=Y^{*}$ in Section 5 .

Much of the paper follows the lines of [10], and we omit proofs if they do not differ much from those in that paper.

We use standard notation such as $B_{X}$ and $S_{X}$ for the unit ball and the unit sphere of a Banach space $X$, and we employ the notation

$$
S_{U}\left(x^{*}, \varepsilon\right)=\left\{x \in U: x^{*}(x)>\sup _{u \in U} x^{*}(u)-\varepsilon\right\}
$$

for the slice of a bounded convex subset $U \subset X$ determined by $x^{*} \in S_{X^{*}}$. In the case of $U=B_{X}$ we omit the index $U$ in the notation above:

$$
S\left(x^{*}, \varepsilon\right)=\left\{x \in B_{X}: x^{*}(x)>1-\varepsilon\right\} .
$$

For $\varepsilon>0$ and $x \in S_{X}$ we consider the weak* slice of the dual ball $B_{X^{*}}$, i.e.,

$$
S(x, \varepsilon)=\left\{x^{*} \in B_{X^{*}}: x^{*}(x)>1-\varepsilon\right\},
$$

as a particular case of a slice. When we need to stress in what space the slice is considered we use notation like $S\left(X, x^{*}, \varepsilon\right)$ or $S\left(X^{*}, x, \varepsilon\right)$. The symbol ex $C$ stands for the set of extreme points of a set $C$. In this paper we deal with real Banach spaces although our results extend to the complex case with minor modifications. 
2. Basic descriptions of the generalised Daugavet property. In this section we collect generalisations of characterisations of the standard Daugavet property to the setting of $\operatorname{DPr}(Y)$. Let us start with a simple lemma about slices; cf. [9, Lemma 2.1].

Lemma 2.1. Let $X$ be any Banach space and $Y \subset X^{*}$ a norming subspace. Let $y \in S_{X}, x_{0}^{*} \in S_{Y}$ and $\varepsilon \in(0,1)$. Assume that there is some $x \in S\left(x_{0}^{*}, \varepsilon / 8\right)$ such that $\|x+y\|>2-\varepsilon / 8$. Then there is an $x_{1}^{*} \in S_{Y}$ such that $S\left(x_{1}^{*}, \varepsilon / 8\right) \subset S\left(x_{0}^{*}, \varepsilon\right)$ and $\|e+y\|>2-\varepsilon$ for all $e \in S\left(x_{1}^{*}, \varepsilon / 8\right)$.

Proof. Since $\|x+y\|>2-\varepsilon / 8$ there is an $x^{*} \in S_{Y}$ such that $x^{*}(x+y)>$ $2-\varepsilon / 8$. Then

$$
x^{*}(x)>1-\frac{\varepsilon}{8} \quad \text { and } \quad x^{*}(y)>1-\frac{\varepsilon}{8} .
$$

Define $x_{1}^{*} \in S_{X^{*}}$ by

$$
x_{1}^{*}=\frac{x_{0}^{*}+x^{*}}{\left\|x_{0}^{*}+x^{*}\right\|} ;
$$

we remark that

$$
\left\|x_{0}^{*}+x^{*}\right\| \geq\left(x_{0}^{*}+x^{*}\right) x>2-\frac{\varepsilon}{4} .
$$

Then for every $e \in S\left(x_{1}^{*}, \varepsilon / 8\right)$ we have

so

$$
\left(x_{0}^{*}+x^{*}\right)(e)>\left(1-\frac{\varepsilon}{8}\right)\left(2-\frac{\varepsilon}{4}\right),
$$

$$
x_{0}^{*}(e)>1-2 \frac{\varepsilon}{8}-\frac{\varepsilon}{4}+\frac{\varepsilon}{8} \frac{\varepsilon}{4}>1-\frac{\varepsilon}{2},
$$

i.e., $e \in S\left(x_{0}^{*}, \varepsilon\right)$, and the inclusion $S\left(x_{1}^{*}, \varepsilon / 8\right) \subset S\left(x_{0}^{*}, \varepsilon\right)$ is proved. Further, (2.2) implies that $x^{*}(e)>1-\varepsilon / 2$, which together with (2.1) means that $\|e+y\| \geq x^{*}(e+y)>2-\varepsilon$.

The following result is the analogue of [9, Lemma 2.2].

TheOREM 2.2. If $Y$ is a norming subspace of $X^{*}$, then the following assertions are equivalent.

(i) $X$ has the Daugavet property with respect to $Y$.

(ii) For every $x \in S_{X}$, for every $\varepsilon>0$, and for every $y^{*} \in S_{Y}$ there is some $y \in S\left(y^{*}, \varepsilon\right)$ such that

$$
\|x+y\| \geq 2-\varepsilon .
$$

(iii) For every $x \in S_{X}$, for every $\varepsilon>0$, and for every $y^{*} \in S_{Y}$ there is a slice $S\left(y_{1}^{*}, \varepsilon_{1}\right) \subset S\left(y^{*}, \varepsilon\right)$ with $y_{1}^{*} \in S_{Y}$ such that (2.3) holds for every $y \in S\left(y^{*}, \varepsilon_{1}\right)$.

Proof. The implication (iii) $\Rightarrow$ (ii) is obvious; the implication (ii) $\Rightarrow$ (iii) follows from Lemma 2.1. What remains to prove is the equivalence (i) $\Leftrightarrow(i i)$. 
Let us start with (i) $\Rightarrow\left(\right.$ ii). Fix some $x \in S_{X}, x^{*} \in S_{Y}$ and $\varepsilon>0$ and consider the operator $T: X \rightarrow X, T:=x^{*} \otimes x$. According to (1.1), $\|\mathrm{Id}+T\|=2$, so there is a $y \in S_{X}$ such that $\|y+T y\| \geq 2-\varepsilon / 2$ and $x^{*}(y) \geq 0$. Substituting the value of $T y$ we obtain

$$
\left\|y+x^{*}(y) x\right\| \geq 2-\varepsilon / 2,
$$

which means that $x^{*}(y) \geq 1-\varepsilon / 2$ (i.e., $y \in S\left(x^{*}, \varepsilon\right)$ ) and

$$
\|y+x\| \geq\left\|y+x^{*}(y) x\right\|-\left|x^{*}(y)-1\right| \geq 2-\varepsilon,
$$

which proves the desired implication.

For the converse implication (ii) $\Rightarrow$ (i) consider an operator $T: X \rightarrow X$, $T=x^{*} \otimes x$, where $x \in X$ and $x^{*} \in Y$. Since the validity of (1.1) for $T$ implies (1.1) for all operators of the form $a T$ with $a>0$, it is sufficient to consider the case of $\|T\|=1$, and the representation $T=x^{*} \otimes x$ can be taken in such a way that $x \in S_{X}$ and $x^{*} \in S_{Y}$. Due to (ii), for every $\varepsilon>0$ there is a $y \in S\left(x^{*}, \varepsilon\right)$ satisfying $(2.3)$. Then

$$
\begin{aligned}
\|\operatorname{Id}+T\| & \geq\|y+T y\|=\left\|y+x^{*}(y) x\right\| \\
& \geq\|y+x\|-\left|x^{*}(y)-1\right| \geq(2-\varepsilon)-\varepsilon,
\end{aligned}
$$

which by arbitrariness of $\varepsilon$ means that $\|\mathrm{Id}+T\|=2$.

3. A useful tool: convex combinations of slices. This section deals with a technical device that will be useful in the proof of Theorem 4.8.

Definition 3.1. Let $Y$ be a norming subspace of $X^{*}$, and let $U \subset X$ be convex and bounded. A subset $V \subset U$ is called a quasi- $\sigma_{U}(X, Y)$ neighbourhood if it is a finite convex combination of slices of $U$ generated by elements of $Y$; i.e., there are $\lambda_{k} \geq 0, k=1, \ldots, n$, with $\sum_{k=1}^{n} \lambda_{k}=1$ and slices $S_{1}, \ldots, S_{n} \subset U$ generated by elements of $Y$ such that $\lambda_{1} S_{1}+\cdots+\lambda_{n} S_{n}=V$.

The following lemma is known for the ordinary weak topology (see [2, Lemme 5.3]; it was rediscovered in [13]). The $\sigma(X, Y)$-version proof coincides almost word-for-word with the original one.

LEMMA 3.2. Under the conditions of the above definition every relatively $\sigma(X, Y)$-open subset $A \subset U$ contains a quasi- $\sigma_{U}(X, Y)$ neighbourhood.

The next theorem and its corollary were essentially proved by Shvidkoy [13]. He considered the ordinary Daugavet property, but the proof in the general case is virtually the same.

TheOREm 3.3. Let $Y$ be a norming subspace of $X^{*}$ and $X \in \operatorname{DPr}(Y)$. Then for every $\varepsilon>0$, every $x \in S_{X}$ and every quasi- $\sigma_{B_{X}}(X, Y)$ neighbourhood $V$ there exists an element $v \in V$ such that $\|v+x\| \geq 2-\varepsilon$.

Proof. Let $V=\sum_{k=1}^{n} \lambda_{k} S_{k}$ be a representation of $V$ as a convex combination of slices. Using repeatedly (ii) of Theorem 2.2 one can construct 
$x_{k} \in S_{k}$ such that

$$
\left\|\left(x+\sum_{j<k} \lambda_{j} x_{j}\right)+\lambda_{k} x_{k}\right\| \geq\left\|x+\sum_{j<k} \lambda_{j} x_{j}\right\|+\lambda_{k}-\varepsilon / n .
$$

Then $v=\sum_{k=1}^{n} \lambda_{k} x_{k}$ will be the element of $V$ we need.

CorollarY 3.4. If $Y$ is a norming subspace of $X^{*}$ and $X$ has the Daugavet property with respect to $Y$, then for every $x \in S_{X}$, for every $\varepsilon>0$, and for every $\sigma(X, Y)$-open subset $U \subset X$ intersecting $B_{X}$ there is some $y \in U \cap B_{X}$ such that $\|x+y\| \geq 2-\varepsilon$.

4. Narrow operators with respect to a norming subspace. We will eventually study subspaces satisfying a certain smallness condition called "poverty"; this will be dual to the notion of a "rich" subspace from [10]. The latter class is defined by the requirement that the canonical quotient map is "narrow". This section deals with such operators.

First we will recall and modify some definitions from [10]. Let $X, E$ be Banach spaces.

Definition 4.1. An operator $T \in L(X, E)$ is said to be a strong Daugavet operator if for any two elements $x, y \in S_{X}$ and for every $\varepsilon>0$ there is an element $z \in S_{X}$ such that $\|z+x\|>2-\varepsilon$ and $\|T z-T y\|<\varepsilon$. We denote the class of all strong Daugavet operators on $X$ by $\mathcal{S D}(X)$.

Corollary 3.4 shows that if $X \in \operatorname{DPr}(Y)$ then every $T \in L(X, E)$ of the form $T=f \otimes e$, where $e \in E$ and $f \in Y$, is a strong Daugavet operator, and conversely, thanks to Theorem 2.2 , if every $f \in Y \subset X^{*}=L(X, \mathbb{R})$ is strongly Daugavet, then $X$ has the Daugavet property with respect to $Y$.

There is an obvious connection between strong Daugavet operators and the Daugavet equation (cf. [10, Lemma 3.2]).

Lemma 4.2. If $T: X \rightarrow X$ is a strong Daugavet operator, then $T$ satisfies the Daugavet equation (1.1).

Definition 4.3. Let $X \in \operatorname{DPr}(Y)$. An operator $T \in L(X, E)$ is said to be narrow with respect to $Y$ (or $Y$-narrow for short) if for every $x, e \in S_{X}$, $\varepsilon>0$ and every slice $S \subset B_{X}$ generated by an element of $Y$ and containing $e$ there is an element $v \in S$ such that $\|x+v\|>2-\varepsilon$ and $\|T v-T e\|<\varepsilon$. We denote the class of all $Y$-narrow operators on $X$ by $\mathcal{N A \mathcal { R }}_{Y}(X)$.

The notations $\mathcal{S D}(X)$ and $\mathcal{N A R}_{Y}(X)$ do not mention the range space $E$ because the corresponding definitions do not actually depend on the values of $T$, but only on the norms of those values, i.e., these are not properties of the operator $T$ itself, but just of the seminorm $x \mapsto\|T(x)\|$ on $X$. For more about this ideology see [10]. 
The following statement is a complete analogue of [10, Lemma 3.10(a)], so we omit the proof.

Lemma 4.4. Let $T \in \mathcal{N A}_{\mathcal{A}}(X)$. Let $S_{1}, \ldots, S_{n} \subset B_{X}$ be a finite collection of slices generated by elements of $Y$, and let $U \subset B_{X}$ be a convex combination of these slices, i.e., there are $\lambda_{k} \geq 0$ with $\sum_{k=1}^{n} \lambda_{k}=1$ such that $\lambda_{1} S_{1}+\cdots+\lambda_{n} S_{n}=U$. Then for every $\varepsilon>0$, every $x_{1} \in S_{X}$ and every $w \in U$ there exists an element $u \in U$ such that $\left\|u+x_{1}\right\|>2-\varepsilon$ and $\|T(w-u)\|<\varepsilon$.

Let us recall an operation with operators that was introduced in [10]. For operators $T_{1}: X \rightarrow E_{1}$ and $T_{2}: X \rightarrow E_{2}$ define

$$
T_{1} \tilde{+} T_{2}: X \rightarrow E_{1} \oplus_{1} E_{2}, \quad x \mapsto\left(T_{1} x, T_{2} x\right) ;
$$

i.e.,

$$
\left\|\left(T_{1} \tilde{+} T_{2}\right) x\right\|=\left\|T_{1} x\right\|+\left\|T_{2} x\right\| .
$$

Remark 4.5. Let $X, E$ be Banach spaces, $Y \subset X^{*}$ be a norming subspace, and $T \in L(X, E)$. If $T \tilde{+} y^{*} \in \mathcal{S D}(X)$ for every $y^{*} \in Y$, then $T \in \mathcal{N A R}_{Y}(X)$. In the setting of $Y=X^{*}$ this was actually given as the definition of a narrow operator in [10], and our definition was stated as an equivalent condition in Lemma 3.10 of [10].

We now introduce a class of operators that turn out to be $Y$-narrow; they correspond to the strong Radon-Nikodým operators for $Y=X^{*}$, which contain the weakly compact operators. We need two technical definitions.

Definition 4.6. Let $X, E$ be Banach spaces, $F \subset E^{*}$ be a norming subspace and $\varepsilon>0$. A point $e$ of a convex subset $A \subset E$ is said to be an $(F, \varepsilon)$-denting point if there is a functional $f \in S_{F}$ and a $\delta>0$ such that $\|e-a\|<\varepsilon$ whenever $a \in A$ satisfies the condition $f(a)>f(e)-\delta$. We say that $A \subset E$ is $F$-dentable if for every $\varepsilon>0$ the set $A$ is contained in the closed convex hull of its $(F, \varepsilon)$-denting points. An operator $T \in L(X, E)$ is said to be $F$-dentable if $T\left(B_{X}\right)$ is $F$-dentable. An $E^{*}$-dentable operator is called dentable.

Definition 4.7. An operator $T \in L(X, E)$ is said to be hereditarily $F$-dentable if for every $x^{*} \in X^{*}$ the operator

$$
T \tilde{+} x^{*}: X \rightarrow E \oplus_{1} \mathbb{R}, \quad x \mapsto\left(T x, x^{*} x\right),
$$

is $\widetilde{F}$-dentable, where $\widetilde{F}$ consists of all functionals $(f, \beta): E \oplus_{1} \mathbb{R} \rightarrow \mathbb{R}$, with $f \in F$ and $\beta \in \mathbb{R}$, of the form $(f, \beta)((e, t))=f(e)+\beta t$.

We remark that every strong Radon-Nikodým operator and in particular every weakly compact operator is hereditarily dentable, by well-known geometric characterisations of sets with the RNP [4, Chap. 3]. 
Theorem 4.8. If $X \in \operatorname{DPr}(Y), T: X \rightarrow E$ is a hereditarily $F$-dentable operator and $T^{*}(F) \subset Y$, then $T$ is $Y$-narrow.

Proof. According to Remark 4.5 it is sufficient to prove that $\widetilde{T}=T \tilde{+} x^{*} \in$ $\mathcal{S D}(X)$ for every $x^{*} \in Y$. Fix $x, z \in S_{X}$ and $\varepsilon>0$. By Definition 4.1 , to prove the theorem we have to find an element $v \in B_{X}$ such that $\|x+v\|>2-\varepsilon$ and $\|\widetilde{T}(z-v)\|<\varepsilon$.

Since $\widetilde{T}\left(B_{X}\right)$ is $\widetilde{F}$-dentable there are $\lambda_{k} \geq 0$ with $\sum_{k=1}^{n} \lambda_{k}=1$ and $(\widetilde{F}, \varepsilon / 2)$-denting points $e_{1}, \ldots, e_{n} \in T\left(B_{X}\right)$ such that

$$
\left\|T z-\sum_{k=1}^{n} \lambda_{k} e_{k}\right\|<\varepsilon / 2 .
$$

By the definition of an $\widetilde{F}$-denting point there are slices $S_{k}=S_{\widetilde{T}\left(B_{X}\right)}\left(f_{k}, \varepsilon_{k}\right)$ of $T\left(B_{X}\right)$ generated by elements of $\widetilde{F}$ such that $e_{k} \in S_{k}$ and the diameter of each of the $S_{k}$ is less than $\varepsilon / 2$. Define $W:=\sum_{k=1}^{n} \lambda_{k} S_{k}$. Since $\operatorname{dist}(T z, W)<$ $\varepsilon / 2$ and $\operatorname{diam} W<\varepsilon / 2$ we have

$$
\|T z-w\|<\varepsilon \quad \text { for every } w \in W .
$$

Set $y_{k}^{*}:=\widetilde{T}^{*} f_{k}$. By assumption, $y_{k}^{*} \in Y$. Consider the slices $V_{k}=\left\{v \in B_{X}\right.$ : $\left.y_{k}^{*}(x)>\varepsilon_{k}\right\}$ and the quasi- $\sigma_{B_{X}}(X, Y)$ neighbourhood $V:=\sum_{k=1}^{n} \lambda_{k} V_{k}$. Since $\widetilde{T}\left(V_{k}\right) \subset S_{k}$ and consequently $\widetilde{T}(V) \subset W,(4.1)$ implies that

$$
\|T z-T v\|<\varepsilon \quad \text { for every } v \in V .
$$

It remains to apply Theorem 3.3 to get a $v \in V$ with $\|x+v\|>2-\varepsilon$.

Corollary 4.9. A weak*-weakly continuous operator on a dual space $X^{*} \in \operatorname{DPr}(X)$ is $X$-narrow.

Proof. It is clear that such an operator is weakly compact. The hereditary dentability of a weakly compact operator $T: X^{*} \rightarrow E$ has been mentioned in the above remark; it remains to observe that $T^{*}\left(E^{*}\right) \subset X$ if $T$ is weak*weakly continuous.

Note that a weakly compact adjoint operator $S^{*}: X^{*} \rightarrow V^{*}$ is weak*weakly continuous, i.e., $S^{* *}\left(V^{* *}\right) \subset X$; cf. [6, Section VI.4].

5. Rich and poor subspaces. In [10] we introduced rich subspaces $Z \subset X$, building on work in [8] and [12]. We showed that this condition is equivalent to saying that every superspace $Z \subset \widetilde{Z} \subset X$ has the Daugavet property; the latter property was called wealth in [10]. We now extend and dualise these ideas.

5.1. Richness. The next proposition shows a kind of stability of the Daugavet property when one passes from the original space to a "big" subspace. 
Lemma 5.1. Let $X \in \operatorname{DPr}(Y)$. Then for every $x \in S_{X}$, every $\varepsilon>0$ and every separable subspace $V \subset Y$ there is an $x^{*} \in S_{X^{*}}$ such that $x^{*}(x) \geq 1-\varepsilon$ and $\left\|x^{*}+f\right\|=1+\|f\|$ for all $f \in V$.

Proof. Consider a dense sequence $\left(f_{n}\right)_{n=1}^{\infty} \subset V$ in which every element is repeated infinitely many times. Applying (v) of Theorem 2.2 to the slice $S(x, \varepsilon)$ of $B_{X^{*}}$ and to $f_{1}$ and then applying it step-by-step to $f_{n}$ and to the slices obtained in the previous steps, we construct a sequence of closed slices $\bar{S}(x, \varepsilon) \supset \bar{S}\left(x_{1}, \varepsilon_{1}\right) \supset \bar{S}\left(x_{2}, \varepsilon_{2}\right) \supset \cdots$ with $\varepsilon_{n}<1 / n$ such that $\left\|x^{*}+f_{n}\right\| \geq$ $2-\varepsilon_{n-1}$ for all $x^{*} \in S\left(x_{n}, \varepsilon_{n}\right)$. By $w^{*}$-compactness of all $\bar{S}\left(x_{n}, \varepsilon_{n}\right)$, there is a point $x^{*} \in \bigcap_{n=1}^{\infty} \bar{S}\left(x_{n}, \varepsilon_{n}\right) \subset \bar{S}(x, \varepsilon)$. This is exactly the point we need.

Proposition 5.2. Let $X \in \operatorname{DPr}(Y)$, and let $Z \subset X$ be a subspace such that $Z^{\perp}$ is a separable subspace of $Y$. Then $Z \in \operatorname{DPr}\left(\left.Y\right|_{Z}\right)$.

Proof. Let $z \in S(Z)$, and let

$$
S=\left\{z^{*} \in Z^{*}=X^{*} / Z^{\perp}:\left\|z^{*}\right\| \leq 1, z^{*}(z) \geq 1-\varepsilon\right\}
$$

be a slice of $B_{Z^{*}}$. Fix a $\left[z^{*}\right] \in S\left(Y / Z^{\perp}\right)$. We have to prove the existence of an $\left[x^{*}\right] \in S$ such that $\left\|x^{*}+z^{*}\right\|=2$. Applying Lemma 5.1 with $x=z$ and $V=\operatorname{lin}\left(\left\{z^{*}\right\} \cup Z^{\perp}\right)$ we obtain an $x^{*} \in S_{X^{*}}$ such that $x^{*}(z) \geq 1-\varepsilon$ and

$$
\left\|x^{*}+f\right\|=1+\|f\| \quad \text { for all } f \in V .
$$

Then $\left[x^{*}\right] \in S$ and

$$
\begin{aligned}
\left\|\left[x^{*}+z^{*}\right]\right\| & =\inf _{f \in Z^{\perp}}\left\|x^{*}+z^{*}+f\right\| \\
& =\inf _{f \in Z^{\perp}}\left(1+\left\|z^{*}+f\right\|\right)=1+\left\|\left[z^{*}\right]\right\|=2 .
\end{aligned}
$$

If a subspace $Z \subset X$ satisfies the conditions of the proposition above then so do all the subspaces of $X$ containing $Z$. Hence $Z$ has the property that $\widetilde{Z} \in \operatorname{DPr}\left(\left.Y\right|_{\widetilde{Z}}\right)$ for every subspace $\widetilde{Z} \subset X$ containing $Z$. Let us formalise this property.

Definition 5.3. Let $X \in \operatorname{DPr}(Y)$. A subspace $Z \subset X$ is said to be wealthy with respect to $Y$ if $\widetilde{Z} \in \operatorname{DPr}\left(\left.Y\right|_{\widetilde{Z}}\right)$ for every subspace $\widetilde{Z} \subset X$ containing $Z$.

Thus Proposition 5.2 can be rephrased by saying that $Z \subset X$ is wealthy with respect to $Y$ if $Z^{\perp}$ is separable and $X \in \operatorname{DPr}(Y)$.

The main result of this subsection is a characterisation of $Y$-wealthy subspaces through $Y$-narrow operators, analogous to [10, Theorem 5.12].

Definition 5.4. Let $X \in \operatorname{DPr}(Y)$. A subspace $Z \subset X$ is said to be rich with respect to $Y$ if the quotient map $q: X \rightarrow X / Z$ is a $Y$-narrow operator.

It turns out that the following theorem holds. 
Theorem 5.5. Let $X$ be a Banach space and $Y$ be a norming subspace of $X^{*}$ such that $X \in \operatorname{DPr}(Y)$. Then for a subspace $Z \subset X$ the following properties are equivalent:

(i) $Z$ is wealthy with respect to $Y$.

(ii) $Z$ is rich with respect to $Y$.

The proof is very similar to the proof of [10, Theorem 5.12].

5.2. Poverty as a dual property to richness

Definition 5.6. Let $X \in \mathrm{DPr}$. A subspace $Z \subset X$ is said to be poor if $X / \widetilde{Z} \in \mathrm{DPr}$ for every subspace $\widetilde{Z} \subset Z$.

Our study of poor subspaces uses duality, so let us start with a very simple observation that we state as a proposition for easy reference.

Proposition 5.7. A Banach space $X$ has the Daugavet property if and only if $X^{*} \in \operatorname{DPr}(X)$. Hence, a subspace $Z$ of a space $X$ with the Daugavet property is poor if and only if for every subspace $\widetilde{Z} \subset Z$ its dual $(X / \widetilde{Z})^{*}=\widetilde{Z}^{\perp}$ has the Daugavet property with respect to $X / \widetilde{Z}$.

Now we are ready to give the basic characterisations of poverty.

Theorem 5.8. Let $X \in$ DPr. For a subspace $Z \subset X$ the following conditions are equivalent.

(i) $Z$ is poor.

(ii) $X / \widetilde{Z} \in \operatorname{DPr}$ for every subspace $\widetilde{Z} \subset Z$ with $\operatorname{codim}_{Z} \widetilde{Z} \leq 2$.

(iii) $Z^{\perp}$ is a subspace of $X^{*}$ that is rich with respect to $X$.

(iv) For every $x^{*}, e^{*} \in S_{X^{*}}, \varepsilon>0$ and for every $x \in S_{X}$ such that $e^{*}(x)>1-\varepsilon$ there is an element $v^{*} \in B_{X^{*}}$ with the following properties: $v^{*}(x)>1-\varepsilon,\left\|x^{*}+v^{*}\right\|>2-\varepsilon$ and $\left\|\left.\left(e^{*}-v^{*}\right)\right|_{Z}\right\|<\varepsilon$; that is, the quotient map from $X^{*}$ onto $X^{*} / Z^{\perp}$ is narrow with respect to $X$.

Proof. (i) $\Rightarrow$ (ii) follows immediately from the definition of poor subspaces.

Let us prove (ii) $\Rightarrow$ (i). According to Proposition 5.7, we have to prove that for every subspace $Z_{1} \subset Z$, its dual $Z_{1}^{\perp}$ has the Daugavet property with respect to $X / Z_{1}$. Fix $Z_{1} \subset Z$. Applying (ii) of Theorem 2.2 we see that for every $x^{*} \in S_{Z_{1}^{\perp}}, \varepsilon>0$ and every $[x] \in S_{X / Z_{1}}$ we have to find $y^{*} \in S_{Z_{1}^{\perp}}$ such that $y^{*}([x]) \geq 1-\varepsilon$ and $\left\|x^{*}+y^{*}\right\| \geq 2-\varepsilon$. Since $[x] \in S_{X / Z_{1}}$, there exists $z^{*} \in S_{Z_{1}^{\perp}}$ such that $z^{*}([x])=1$. Define $\widetilde{Z}=Z \cap \operatorname{ker} x^{*} \cap$ ker $z^{*}$. Evidently, $\widetilde{Z}$ is a subspace of $Z$ with $\operatorname{codim}_{Z} \widetilde{Z} \leq 2$ and $Z_{1} \subset \widetilde{Z}$. Also note that

$$
1=\left\|[x]_{X / Z_{1}}\right\| \geq\|[x]\|_{X / \widetilde{Z}} \geq z^{*}([x])=1,
$$


which implies $[x] \in S_{X / \widetilde{Z}}$. By our assumption $\widetilde{Z}^{\perp}$ has the Daugavet property with respect to $X / \widetilde{Z}$, and hence for $x^{*} \in S_{\widetilde{Z}^{\perp}}$ and $[x] \in S_{X / \widetilde{Z}}$ there is $y^{*} \in$ $S_{\widetilde{Z}^{\perp}}$ such that $y^{*}([x]) \geq 1-\varepsilon$ and $\left\|x^{*}+y^{*}\right\| \geq 2-\varepsilon$. Then $y^{*} \in S_{\widetilde{Z}^{\perp}} \subset S_{Z_{1}^{\perp}}$, and it meets all the requirements.

Now we will prove that (ii) $\Leftrightarrow$ (iii). Theorem 5.5 implies that (iii) holds if and only if $Z^{\perp}$ is a subspace of $X^{*}$ that is wealthy with respect to $X$; and this is equivalent to the claim that for every $x^{*}, y^{*} \in S_{X^{*}}$ the space $W=\operatorname{lin}\left(Z^{\perp} \cup\left\{x^{*}, y^{*}\right\}\right)$ has the Daugavet property with respect to $X / W_{\perp}$ (cf. [10, Lemma 5.6(iii)]. But for a space $\widehat{Z} \supset Z^{\perp}$ the existence of $x^{*}, y^{*} \in S_{X^{*}}$ such that $W=\operatorname{lin}\left(Z^{\perp} \cup\left\{x^{*}, y^{*}\right\}\right)$ is equivalent to the existence of a space $\widetilde{Z} \subset Z$ such that $W=\widetilde{Z}^{\perp}$ and $\operatorname{codim}_{Z} \widetilde{Z} \leq 2$. Thus we see that (iii) is equivalent to the claim that $\widetilde{Z}^{\perp} \in \operatorname{DPr}(X / \overline{\widetilde{Z}})$ for every subspace $\widetilde{Z} \subset Z$ with $\operatorname{codim}_{Z} \widetilde{Z} \leq 2$, which is equivalent to (ii) according to Proposition 5.7.

The remaining equivalence (iii) $\Leftrightarrow$ (iv) is just a reformulation of the definition of a rich subspace.

As a corollary we can give a proof of the following theorem of Shvidkoy [13].

Corollary 5.9. Let $X \in \mathrm{DPr}$ and let $Z$ be a reflexive subspace of $X$. Then the quotient space $X / Z$ also has the Daugavet property.

Proof. Since every subspace of a reflexive space is also reflexive, the statement of this corollary is equivalent to the claim that every reflexive subspace $Z$ of $X \in \mathrm{DPr}$ is poor. According to Theorem 5.8, it is sufficient to prove that $Z^{\perp}$ is a subspace of $X^{*}$ that is rich with respect to $X$, i.e., that the quotient map $q: X^{*} \rightarrow X^{*} / Z^{\perp}$ is an $X$-narrow operator. As $X^{*} / Z^{\perp}$ is isometric to $Z^{*}$, which is reflexive, this follows from Corollary 4.9 .

6. Applications to the geometry of $C(K)$ and $L_{1}$. For a compact Hausdorff space $K$ denote by $M(K)$ the dual space of $C(K)$, i.e., $M(K)$ is the Banach space of all (not necessarily positive) finite regular Borel signed measures on $K$. (In the following, all measures on $K$ will be tacitly assumed to be finite regular Borel measures.) We are going to prove a theorem which gives a characterisation of operators on $M(K)$ that are narrow with respect to $C(K)$. For this theorem we will need the following lemma in which $\partial A$ denotes the boundary of a set $A \subset K$.

Lemma 6.1. Let $K$ be compact, $f \in C(K)$, and $\mu$ be some positive measure on $K$. Then for every $\varepsilon>0$ there exists a step function $\tilde{f}=\sum_{k=1}^{n} \beta_{k} \chi_{A_{k}}$ on $K$ such that $\mu\left(\partial A_{k}\right)=0$ for $k=1, \ldots, n, A_{1} \cup \cdots \cup A_{n}=K$ and $\|f-\widetilde{f}\|_{\infty}<\varepsilon$. 
Proof. Since the image measure $\nu=\mu \circ f^{-1}$ on $\mathbb{R}$ has at most countably many atoms, it is possible to cover $f(K)$ by finitely many half-open intervals $I_{k}=\left(\beta_{k-1}, \beta_{k}\right]$ of length $<\varepsilon$ such that $\nu\left(\left\{\beta_{0}, \ldots, \beta_{n}\right\}\right)=0$. Let $A_{k}=$ $f^{-1}\left(I_{k}\right)$; then $\widetilde{f}=\sum_{k=1}^{n} \beta_{k} \chi A_{k}$ works.

THEOREM 6.2. Let $K$ be a perfect compact Hausdorff space. An operator $T$ on $M(K)$ is narrow with respect to $C(K)$ if and only if for every open subset $U \subset K$, for any two probability measures $\pi_{1}, \pi_{2}$ on $U$ and for every $\varepsilon>0$ there is a probability measure $\nu$ on $U$ such that $\left\|T\left(\nu-\pi_{1}\right)\right\|<\varepsilon$ and $\left\|\pi_{2}-\nu\right\|>2-\varepsilon$.

Proof. We first prove the "only if" part. By the definition of a narrow operator (Definition 4.3), for every $x, e \in S_{M(K)}, \varepsilon>0$ and every weak* slice $S$ of $B_{M(K)}$ containing $e$, there exists $v \in S$ such that $\|x+v\|>2-\varepsilon$ and $\|T(e-v)\|<\varepsilon$. Fix $\varepsilon_{1}>0$ and let $x=-\pi_{2}$ and $e=\pi_{1}$. Since $U$ is open and $\pi_{1}(U)=1$, we can find $f \in C(K)$ taking values in [0,1] with supp $f \subset U$ and $\int f d \pi_{1}>1-\varepsilon_{1}$. By Definition 4.3 there exists $\widetilde{\nu} \in S_{M(K)}$ such that the following inequalities hold:

$$
\int f d \widetilde{\nu}>1-\varepsilon_{1}, \quad\left\|T\left(\widetilde{\nu}-\pi_{1}\right)\right\|<\varepsilon_{1}, \quad\left\|\pi_{2}-\widetilde{\nu}\right\|>2-\varepsilon_{1} .
$$

Let $\widehat{\nu}=\left.\widetilde{\nu}^{+}\right|_{U}$. Using the properties of $f$ we have $\|\widetilde{\nu}-\widehat{\nu}\|<2 \varepsilon_{1}$ and thus $1-3 \varepsilon_{1}<\|\widehat{\nu}\| \leq 1+2 \varepsilon_{1}, \quad\left\|T\left(\widehat{\nu}-\pi_{1}\right)\right\|<\varepsilon_{1}(1+2\|T\|), \quad\left\|\pi_{2}-\widehat{\nu}\right\|>2-3 \varepsilon_{1}$. Hence for $\nu=\widehat{\nu} /\|\widehat{\nu}\|$ we have $\|\nu-\widehat{\nu}\|=|1-\|\widehat{\nu}\||<3 \varepsilon_{1}$, and consequently

$$
\left\|\pi_{2}-\nu\right\| \geq\left\|\pi_{2}-\widehat{\nu}\right\|-\|\nu-\widehat{\nu}\|>2-3 \varepsilon_{1}-3 \varepsilon_{1}=2-6 \varepsilon_{1},
$$

and

$$
\left\|T\left(\nu-\pi_{1}\right)\right\| \leq\left\|T\left(\widehat{\nu}-\pi_{1}\right)\right\|+\|T(\widehat{\nu}-\nu)\|<(1+5\|T\|) \varepsilon_{1} .
$$

Then taking $\varepsilon_{1}=\min \{\varepsilon / 6, \varepsilon /(1+5\|T\|)\}$ completes the proof of the "only if" part.

Now consider the "if" part. Given $\mu_{1}, \mu_{2} \in S_{M(K)}, \varepsilon>0$ and a weak* slice $S$ of $B_{M(K)}$ containing $\mu_{1}$, we have to find $\nu \in S$ such that $\left\|\mu_{2}+\nu\right\|>2-\varepsilon$ and $\left\|T\left(\mu_{1}-\nu\right)\right\|<\varepsilon$. Since one can wiggle the slice $S$ a bit, there is, by Lemma 6.1 , no loss of generality in replacing $S$ by a slice generated by a function of the form $f=\sum_{k=1}^{n} \beta_{k} \chi_{A_{k}}$, where $A_{1}, \ldots, A_{n}$ are measurable sets with $\left(\left|\mu_{1}\right|+\left|\mu_{2}\right|\right)\left(\bigcup_{k=1}^{n} \partial A_{k}\right)=0$. (Note that in general this new slice will not be relatively weak* open.) On the other hand, using the Hahn decomposition theorem we have $K=\bigcup_{i=1}^{4} B_{i}$, where $B_{1}$ is a set on which $\mu_{1}$ is positive and $\mu_{2}$ is negative, $B_{2}$ is a set on which $\mu_{2}$ is positive and $\mu_{1}$ is negative, and $B_{3}$ (resp. $\left.B_{4}\right)$ is a set where both $\mu_{1}$ and $\mu_{2}$ are positive (resp. negative).

Fix $\varepsilon_{1}>0$ and let $G_{1}$ be an open set such that $G_{1} \supset B_{1}$ and $\left|\mu_{i}\right|\left(G_{1} \backslash B_{1}\right)$ $<\varepsilon_{1}(i=1,2)$. Define $C_{k}=G_{1} \cap A_{k}$ and let $U_{k}=\operatorname{int} C_{k}, k=1, \ldots, n$. 
Clearly $C_{k} \backslash U_{k} \subset \partial A_{k}$, so the $U_{k}$ are open sets with the following properties: $U_{k} \subset C_{k}$ and $\left(\left|\mu_{1}\right|+\left|\mu_{2}\right|\right)\left(C_{k} \backslash U_{k}\right)=0$.

Consider those $U_{k}$ for which $\mu_{1}\left(U_{k} \cap B_{1}\right) \neq 0, \mu_{2}\left(U_{k} \cap B_{1}\right) \neq 0$ and define two probability measures on $U_{k}$ by

$$
\mu_{i, k}=\frac{\left.\mu_{i}\right|_{U_{k} \cap B_{1}}}{\mu_{i}\left(U_{k} \cap B_{1}\right)} \quad(i=1,2) .
$$

By assumption there exists a probability measure $\widehat{\nu}_{k}$ on $U_{k}$ such that

$$
\left\|T\left(\widehat{\nu}_{k}-\mu_{1, k}\right)\right\|<\varepsilon_{1} \text { and }\left\|\mu_{2, k}-\widehat{\nu}_{k}\right\|>2-\varepsilon_{1} .
$$

Define $\nu_{k}=\mu_{1}\left(U_{k} \cap B_{1}\right) \cdot \widehat{\nu}_{k}$. Then we have

$$
\left\|\nu_{k}\right\|=\nu_{k}\left(U_{k}\right)=\mu_{1}\left(U_{k} \cap B_{1}\right), \quad\left\|\left.\mu_{1}\right|_{U_{k}}\right\|-\varepsilon_{1} \leq\left\|\nu_{k}\right\| \leq\left\|\left.\mu_{1}\right|_{U_{k}}\right\|+\varepsilon_{1}
$$

and

$$
\begin{aligned}
\left\|\left.\mu_{2}\right|_{U_{k}}+\nu_{k}\right\| & =\left\|\mu_{2}\left(U_{k} \cap B_{1}\right) \cdot \mu_{2, k}+\left.\mu_{2}\right|_{U_{k} \backslash B_{1}}+\mu_{1}\left(U_{k} \cap B_{1}\right) \cdot \widehat{\nu}_{k}\right\| \\
& \geq\left\|\left|\mu_{2}\left(U_{k} \cap B_{1}\right)\right| \cdot \mu_{2, k}-\left|\mu_{1}\left(U_{k} \cap B_{1}\right)\right| \cdot \widehat{\nu}_{k}\right\|-\left\|\left.\mu_{2}\right|_{U_{k} \backslash B_{1}}\right\| \\
& \geq\left|\mu_{2}\right|\left(U_{k}\right)+\left|\mu_{1}\right|\left(U_{k}\right)-4 \varepsilon_{1}
\end{aligned}
$$

and

$$
\begin{aligned}
\left\|T\left(\nu_{k}-\left.\mu_{1}\right|_{U_{k}}\right)\right\| & \leq\left\|T\left(\mu_{1}\left(U_{k} \cap B_{1}\right) \cdot\left(\widehat{\nu}_{k}-\mu_{1, k}\right)\right)\right\|+\left\|T\left(\left.\mu_{1}\right|_{U_{k} \backslash B_{1}}\right)\right\| \\
& \leq \varepsilon_{1}(1+\|T\|) .
\end{aligned}
$$

For $U_{k}$ with $\mu_{1}\left(U_{k} \cap B_{1}\right)=0$ or $\mu_{2}\left(U_{k} \cap B_{1}\right)=0$, the inequalities (6.1)-(6.3) hold with $\nu_{k}=\left.\mu_{1}\right|_{U_{k} \cap B_{1}}$.

Now define the measure $\mu_{1}^{1}$ by

$$
\left.\mu_{1}^{1}\right|_{U_{k}}=\nu_{k},\left.\quad \mu_{1}^{1}\right|_{K \backslash \bigcup_{k=1}^{n} U_{k}}=\left.\mu_{1}\right|_{K \backslash \bigcup_{k=1}^{n} U_{k}} .
$$

From (6.1)-(6.3) we obtain the following properties of $\mu_{1}^{1}$ :

$$
\left\|\mu_{1}\right\|-n \varepsilon_{1} \leq\left\|\mu_{1}^{1}\right\| \leq\left\|\mu_{1}\right\|+n \varepsilon_{1}, \quad\left|\int f d \mu_{1}^{1}-\int f d \mu_{1}\right| \leq n \varepsilon_{1}
$$

and

$$
\begin{aligned}
\left\|\left.\mu_{2}\right|_{G_{1}}+\left.\mu_{1}^{1}\right|_{G_{1}}\right\| & \geq\left\|\sum_{k=1}^{n}\left(\mu_{2} \mid U_{k}+\nu_{k}\right)\right\|-\left(\left|\mu_{2}\right|+\left|\mu_{1}\right|\right)\left(\bigcup_{k=1}^{n} C_{k} \backslash U_{k}\right) \\
& \geq \sum_{k=1}^{n}\left(\left|\mu_{2}\right|\left(U_{k}\right)+\left|\mu_{1}\right|\left(U_{k}\right)\right)-4 n \varepsilon_{1} \\
& \geq\left|\mu_{1}\right|\left(G_{1}\right)+\left|\mu_{2}\right|\left(G_{1}\right)-(4 n+2) \varepsilon_{1}, \\
\left\|T\left(\mu_{1}^{1}-\mu_{1}\right)\right\| & =\left\|\sum_{k=1}^{n} T\left(\nu_{k}-\left.\mu_{1}\right|_{U_{k}}\right)\right\| \leq(n+n\|T\|) \varepsilon_{1} .
\end{aligned}
$$

Now define $\widetilde{B}_{2}=B_{2} \backslash G_{1}$. Notice that $\widetilde{B}_{2}$ is a set of negativity for $\mu_{1}^{1}$ and a set of positivity for $\mu_{2}$. Following the same lines as above we define 
$G_{2} \supset \widetilde{B}_{2}$ and construct $\mu_{1}^{2} \in M(K)$ such that

$\left|\mu_{2}\right|\left(G_{2} \backslash \widetilde{B}_{2}\right)<\varepsilon_{1}, \quad\left|\mu_{1}^{1}\right|\left(G_{2} \backslash \widetilde{B}_{2}\right)<\varepsilon_{1}, \quad\left\|\mu_{1}^{1}\right\|-n \varepsilon_{1} \leq\left\|\mu_{1}^{2}\right\| \leq\left\|\mu_{1}^{1}\right\|+n \varepsilon_{1}$

and

$$
\begin{aligned}
\left|\int f d \mu_{1}^{2}-\int f d \mu_{1}^{1}\right| & \leq n \varepsilon_{1}, \\
\left\|T\left(\mu_{1}^{2}-\mu_{1}^{1}\right)\right\| & \leq(n+n\|T\|) \varepsilon_{1}, \\
\left\|\left.\mu_{2}\right|_{G_{2}}+\left.\mu_{1}^{2}\right|_{G_{2}}\right\| & \geq\left|\mu_{1}\right|\left(G_{2}\right)+\left|\mu_{2}\right|\left(G_{2}\right)-(4 n+2) \varepsilon_{1} .
\end{aligned}
$$

From (6.4)-(6.6) and the above inequalities we obtain the estimates

$$
1-2 n \varepsilon_{1} \leq\left\|\mu_{1}^{2}\right\| \leq 1-2 n \varepsilon_{1}, \quad\left|\int f d \mu_{1}^{2}-\int f d \mu_{1}\right| \leq 2 n \varepsilon_{1}
$$

and

$$
\begin{aligned}
\left\|T\left(\mu_{1}^{2}-\mu_{1}\right)\right\| & \leq(2 n+2 n\|T\|) \varepsilon_{1}, \\
\left\|\left.\mu_{2}\right|_{G_{1} \cup G_{2}}+\left.\mu_{1}^{2}\right|_{G_{1} \cup G_{2}}\right\| & \geq\left|\mu_{1}\right|\left(G_{1} \cup G_{2}\right)+\left|\mu_{2}\right|\left(G_{1} \cup G_{2}\right)-(8 n+10) \varepsilon_{1} .
\end{aligned}
$$

Finally, the definition of the sets $B_{3}$ and $B_{4}$ implies that

$$
\left\|\mu_{2}+\mu_{1}^{2}\right\| \geq\left\|\mu_{1}\right\|+\left\|\mu_{2}\right\|-(8 n+10) \varepsilon_{1}=2-(8 n+10) \varepsilon_{1} .
$$

Hence for $\varepsilon_{1}$ small enough, the normalized signed measure $\nu=\mu_{1}^{2} /\left\|\mu_{1}^{2}\right\|$ satisfies all the required conditions, which completes the proof of the theorem.

Applying this theorem to the operator $\left.\mu \mapsto \mu\right|_{Z}$ yields, by Theorem 5.8:

Corollary 6.3. Let $K$ be a perfect compact space. A subspace $Z \subset$ $C(K)$ is poor if and only if for every open subset $U \subset K$, for any two probability measures $\pi_{1}, \pi_{2}$ on $U$ and for every $\varepsilon>0$ there is a probability measure $\nu$ on $U$ such that $\left\|\nu-\pi_{1}\right\|_{Z^{*}}<\varepsilon$ and $\left\|\pi_{2}-\nu\right\|>2-\varepsilon$.

For a closed subset $K_{1}$ of $K$ denote by $R_{K_{1}}$ the natural restriction operator $R_{K_{1}}: C(K) \rightarrow C\left(K_{1}\right)$. Note that for an operator $S: E \rightarrow F$ between Banach spaces the following assertions are equivalent, by the (proof of) the open mapping theorem: (i) $S$ is onto; (ii) $S\left(B_{E}\right)$ is not nowhere dense; (iii) 0 is an interior point of $S\left(B_{E}\right)$.

COROLlary 6.4. Let $K$ be a perfect compact Hausdorff space, $K_{1} \subset K$ be a closed subset with non-empty interior, and let $Z$ be a poor subspace of $C(K)$. Then $R_{K_{1}}\left(B_{Z}\right)$ is nowhere dense in $B_{C\left(K_{1}\right)}$.

Proof. Apply Corollary 6.3 with $U=\operatorname{int} K_{1}, \pi_{1}=\pi_{2}$ and a sufficiently small $\varepsilon>0$ to see that $R_{K_{1}}\left(B_{Z}\right)$ cannot contain a ball $r B_{C\left(K_{1}\right)}$ of radius $r>0$.

We now deal with poor subspaces of $L_{1}$. Let $(\Omega, \Sigma, \mu)$ be a finite measure space. Denote by $\Sigma^{+}$the collection of all $A \in \Sigma$ with $\mu(A)>0$.

TheOREM 6.5. Let $(\Omega, \Sigma, \mu)$ be a non-atomic finite measure space. An operator $T$ on $L_{\infty}:=L_{\infty}(\Omega, \Sigma, \mu)$ is narrow with respect to $L_{1}:=L_{1}(\Omega, \Sigma, \mu)$ 
if and only if for every $\Delta \in \Sigma^{+}$and for every $\varepsilon>0$ there is $g \in S_{L_{\infty}}$ such that $g=0$ off $\Delta$ and $\|T g\|<\varepsilon$. Moreover, in the statement above, $g$ can be selected non-negative.

Proof. First we prove the "if" part. By the definition of a narrow operator (Definition 4.3) for every $x, y \in S_{L_{\infty}}$, every $f \in S_{L_{1}}$ such that $\int f \cdot y d \lambda>1-\delta$ (i.e., $y \in S(f, \delta))$ and every $\varepsilon>0$ we have to find $z \in S(f, \delta)$ such that $\|x+z\|>2-\varepsilon$ and $\|T(y-z)\|<\varepsilon$. By the density of the step functions we may assume without loss of generality that there is a partition $A_{1}, \ldots, A_{n}$ of $\Omega$ such that the restrictions of $x, y$ and $f$ to $A_{k}$ are constants, say $a_{k}$, $b_{k}$ and $c_{k}$ respectively. Fix some $\varepsilon_{1}>0$. Since $\|x\|=1$, there exists $k$ such that $\left|a_{k}\right|>1-\varepsilon_{1}$. Let $B \in \Sigma^{+}$be a subset of $A_{k}$ with $\mu(B) \leq \varepsilon_{1}$ and $A_{k} \backslash B \in \Sigma^{+}$. By our assumption there exists $\widehat{z} \in S_{L_{\infty}}$ such that $z \geq 0, z$ is supported on $B$ and $\|T(z)\| \leq \varepsilon_{1}$. Define $\widetilde{z}=y+\left(\operatorname{sign}\left(a_{1}\right)-b_{1}\right) \widehat{z}$. It is easy to see that $\|\widetilde{z}\|=1,\|x+\widetilde{z}\|=2-\varepsilon_{1},\|T(y-\widetilde{z})\|<\varepsilon_{1}$ and $\widetilde{z} \in S\left(f, \delta-\varepsilon_{1}\right)$. To finish the proof of this part it is sufficient to repeat the reasoning from the end of the proof of Theorem 6.2.

Now we consider the "only if" part. Since $T$ is narrow with respect to $L_{1}$, $T$ is also a strong Daugavet operator. Hence as in [10, Theorem 3.5] we can get a function $\widetilde{g} \in S_{L_{\infty}}$ which satisfies all the requirements, except being non-negative. To fix this we argue as in [8, Lemma 1.4] and finally get some non-negative $g$ possessing all the properties listed above.

Again, specialising to the restriction operator $g \in L_{\infty}=\left.\left(L_{1}\right)^{*} \mapsto g\right|_{Z}$ $\in Z^{*}$ we obtain the following characterisation of poor subspaces.

COROllary 6.6. Let $(\Omega, \Sigma, \mu)$ be a non-atomic finite measure space. A subspace $Z \subset L_{1}(\Omega, \Sigma, \mu)$ is poor if and only if for every $\Delta \in \Sigma^{+}$and for every $\varepsilon>0$ there is $g \in S_{L_{\infty}}$ such that $g=0$ off $\Delta$ and $\|g\|_{Z^{*}}<\varepsilon$. Moreover, in the statement above, $g$ can be selected non-negative.

For a subset $A \in \Sigma^{+}$denote by $Q_{A}$ the natural restriction operator $Q_{A}$ : $L_{1}(\Omega, \Sigma, \mu) \rightarrow L_{1}\left(A,\left.\Sigma\right|_{A},\left.\mu\right|_{A}\right)$.

Corollary 6.7. Let $(\Omega, \Sigma, \mu)$ be a non-atomic finite measure space, $A \in \Sigma^{+}$and let $Z$ be a poor subspace of $L_{1}(\Omega, \Sigma, \mu)$. Then $Q_{A}\left(B_{Z}\right)$ is nowhere dense in $B_{L_{1}\left(A,\left.\Sigma\right|_{A}, \mu\right)}$.

Proof. Apply Corollary 6.6 with $\Delta=A$ and a sufficiently small $\varepsilon>0$ to see that $Q_{A}\left(B_{Z}\right)$ cannot contain a ball $r B_{L_{1}(A)}$ of radius $r>0$.

Corollaries 6.4 and 6.7 look very similar. The next definition extracts the significant common feature.

Definition 6.8. Let $X \in \mathrm{DPr}$. A subspace $E \subset X$ is said to be a bank if $E$ contains an isomorphic copy of $\ell_{1}$ and for every poor subspace $Z$ of $X$, 
$q_{E}\left(B_{Z}\right)$ is nowhere dense in $B_{X / E}$ (here $q_{E}$ denotes the natural quotient map $\left.q_{E}: X \rightarrow X / E\right)$. If $E \subset X$ is a bank, then $B_{X / E}$ will be called the asset of $E$.

In this terminology a poor subspace cannot cover a "significant part" of a bank's asset.

Theorem 6.9. Let $X \in \mathrm{DPr}$ and $E \subset X$ be a bank with separable asset. Then $X$ contains a copy of $\ell_{1}$ which is not poor in $X$.

Proof. Let $\left\{e_{n}\right\}_{n \in \mathbb{N}} \subset \frac{1}{2} B_{E}$ be equivalent to the canonical basis of $\ell_{1}$ and let $\left\{x_{n}\right\}_{n \in \mathbb{N}} \subset B_{E}$ be a sequence such that $\left\{q_{E}\left(x_{n}\right)\right\}_{n \in \mathbb{N}}$ is dense in $B_{X / E}$. Then, if one selects a sufficiently small $\varepsilon>0$, the sequence of $u_{n}=$ $e_{n}+\varepsilon x_{n} \in B_{E}$ is still equivalent to the canonical basis of $\ell_{1}$, and the image of this sequence under $q_{E}$ equals $\left\{\varepsilon q_{E}\left(x_{n}\right)\right\}_{n \in \mathbb{N}}$, which is dense in $\varepsilon B_{X / E}$. This means that the closed linear span of $\left\{u_{n}\right\}_{n \in \mathbb{N}}$ is the copy of $\ell_{1}$ we need.

The next theorem is an immediate corollary of Theorem 6.9.

TheOrem 6.10. In every $C(K)$-space with perfect metric compact $K$ and in every separable $L_{1}(\Omega, \Sigma, \mu)$-space with non-atomic $\mu$ there is a subspace isomorphic to $\ell_{1}$ that is not poor.

Proof. Corollary 6.4 implies that if $K$ is a perfect compact and $K_{1} \subset K$ is a proper closed subset with non-empty interior, then $C_{0}\left(K \backslash K_{1}\right):=\{f \in$ $\left.C(K): f(t)=0 \forall t \in K_{1}\right\}$ is a bank with $B_{C\left(K_{1}\right)}$ being its asset. Corollary 6.7 implies that if $(\Omega, \Sigma, \mu)$ is a non-atomic finite measure space and $A \in \Sigma^{+}$, then $L_{1}(\Omega \backslash A)$ is a bank with $B_{L_{1}(A)}$ being its asset. Separability of these assets follows from the separability of the spaces $C(K)$ and $L_{1}(\Omega, \Sigma, \mu)$ considered. It remains to apply Theorem 6.9.

Theorem 6.10 answers Pełczyński's question mentioned in the introduction in the negative since it provides a non-poor $\ell_{1}$-subspace $Z \subset L_{1}[0,1]$. By definition this means that for some subspace $\widetilde{Z} \subset Z, L_{1}[0,1] / \widetilde{Z}$ fails to have the Daugavet property; but $Z$ has the RNP and so does its subspace $\widetilde{Z}$. In fact, by Theorem 5.8 one can choose $\widetilde{Z}$ of codimension $\leq 2$, hence $\widetilde{Z}$ is isomorphic to $\ell_{1}$ as well. Let us sum up these considerations.

COROLlary 6.11. There is a subspace $E \subset L_{1}[0,1]$ that is isomorphic to $\ell_{1}$ and hence has the $R N P$, but $L_{1}[0,1] / E$ does not have the Daugavet property.

\section{Some open questions}

1. Is it true that every separable space with the Daugavet property has an $\ell_{1}$-subspace which is not poor?

2. Can the separability condition in Theorem 6.9 be omitted? 
3. Is it true that every subspace without copies of $\ell_{1}$ of a space with the Daugavet property is poor? We do not even know the answer in the case of $C[0,1]$.

4. Is it true that if $X \in \mathrm{DPr}$ and $Y \subset X$ is a subspace with a separable dual, then the quotient space $X / Y$ also has the Daugavet property? This question also appears in [13].

\section{References}

[1] D. Bilik, V. M. Kadets, R. V. Shvidkoy and D. Werner, Narrow operators and the Daugavet property for ultraproducts, Positivity 9 (2005), 45-62.

[2] J. Bourgain, La propriété de Radon-Nikodym, Publ. Math. Univ. Pierre et Marie Curie 36 (1979).

[3] J. Bourgain and H. P. Rosenthal, Martingales valued in certain subspaces of $L^{1}$, Israel J. Math. 37 (1980), 54-75.

[4] R. D. Bourgin, Geometric Aspects of Convex Sets with the Radon-Nikodym Property, Lecture Notes in Math. 993, Springer, Berlin, 1983.

[5] G. Choquet, Lectures on Analysis, Vol. II, W. A. Benjamin, New York, 1969.

[6] N. Dunford and J. T. Schwartz, Linear Operators. Part 1: General Theory, Interscience Publ., New York, 1958.

[7] V. M. Kadets, N. Kalton, and D. Werner, Remarks on rich subspaces of Banach spaces, Studia Math. 159 (2003), 195-206.

[8] V. M. Kadets and M. M. Popov, The Daugavet property for narrow operators in rich subspaces of $C[0,1]$ and $L_{1}[0,1]$, St. Petersburg Math. J. 8 (1997), 571-584.

[9] V. M. Kadets, R. V. Shvidkoy, G. G. Sirotkin, and D. Werner, Banach spaces with the Daugavet property, Trans. Amer. Math. Soc. 352 (2000), 855-873.

[10] V. M. Kadets, R. V. Shvidkoy, and D. Werner, Narrow operators and rich subspaces of Banach spaces with the Daugavet property, Studia Math. 147 (2001), 269-298.

[11] V. M. Kadets and D. Werner, A Banach space with the Schur and the Daugavet property, Proc. Amer. Math. Soc. 132 (2004), 1765-1773.

[12] A. M. Plichko and M. M. Popov, Symmetric function spaces on atomless probability spaces, Dissertationes Math. 306 (1990).

[13] R. V. Shvydkoy, Geometric aspects of the Daugavet property, J. Funct. Anal. 176 (2000), 198-212.

[14] M. Talagrand, The three-space problem for $L^{1}$, J. Amer. Math. Soc. 3 (1990), 9-29.

Vladimir Kadets and Varvara Shepelska

Department of Mechanics and Mathematics

Kharkov National University

pl. Svobody 4

61077 Kharkov, Ukraine

E-mail: vova1kadets@yahoo.com shepelskaya@yahoo.com
Dirk Werner

Department of Mathematics

Freie Universität Berlin

Arnimallee 6

D-14195 Berlin, Germany

E-mail: werner@math.fu-berlin.de

Received February 11, 2008;

received in final form June 10, 2008 